\title{
Semantic and Contextual Proximities for Informal Learning : The Case Study of Museum Visits
}

\author{
Pierre-Yves Gicquel \\ Heudiasyc CNRS \\ University of Technology of Compiegne \\ Centre de Recherches de Royallieu \\ 60205 COMPIEGNE cedex France
}

\author{
Dominique Lenne \\ Heudiasyc CNRS \\ University of Technology of Compiegne \\ Centre de Recherches de Royallieu \\ 60205 COMPIEGNE cedex France
}

\begin{abstract}
This work aims to instrument situations of museum visit using mobile devices. The main objective is then to adapt activities and interactions seamlessly for the visitor, according to his desires, his physical location and psychological context. This adaptation involves a detailed model of the learning domain and of the visitor's situation as well as the design of adaptation mechanisms to provide information or activities appropriately during the visit. To this end, semantic models of the learning domain (Cultural Heritage) and of the visitor's context are proposed. These models allow the computation of semantic and contextual proximities, which enables to generate learning activities (recommendations, self-assessment games) while assessing the adequation of these activities to the visitor context.
\end{abstract}

\section{General Terms:}

Ubiquitous learning, Semantic web, Museum

\section{Keywords:}

Ubiquitous learning, Semantic proximity, Context, Semantic modelsifx

\section{INTRODUCTION}

The rise of mobile devices has led to a new paradigm of technology enhanced learning (TEL) environments: ubiquitous learning environments. Like many classic TEL environments, ubiquitous learning environments offer a computer based mediation between the learner and the learning domain. However, in ubiquitous environments, this mediation takes place in a specific physical context which partly determines the learning scenario. The learner acts both in the physical and in the digital spaces. Objects in the physical environment, as well as events or processes around the learner are taken into account to provide appropriate information or to propose relevant interactions.

This work focuses on ubiquitous learning in situations such as informal museum visits. An adaptation of semantics formalisms for describing cultural heritage is proposed to offer a rich semantic model of artworks. Ubiquitous learning systems are based on a representation of the learner's context. Taking inspiration from the work of [15], the context is represented according to several contextual spaces, each space being associated with a contextual ontology which formalizes the modalities of contextual interaction for this space (e.g. an event and time ontology for the interaction history context). These spaces construct a link between the contextual knowledge and the learning domain knowledge. Furthermore, these spaces are automatically populated on the basis of the visitor's movements and his interactions with the device. The learning and context models are operationalized using semantic proximities. The context is taken into account to generate activities by means of a computation of contextual proximities. This computation measures the adequacy of the generated activities to the visitor's context.

This paper describes first some related works and approaches for technology enhanced learning in museum. It then details a proposition of semantics models of the domain and context and the computation of semantic and contextual proximities for these models. Finally, it describes the generation of contextualized learning activities permitted by these computations and presents some firsts results.

\section{STATE OF THE ART}

Learning in museum and learning from museum has been a field widely studied over the last decade. Some major considerations have emerged from these studies [5]. At first it is essential to take into account the motivations of the museum visitor: why is he in the museum for, what are his interests? Then in a constructivist learning perspective it is fundamental to take into account the variety of visitors personal histories to provide them the right content [1]. Finally the social role of exchanging with the peers during the visit is essential [4] to construct a shared understanding of the exhibit.

Various works have been proposed to instrument museum visits either for school visits or for informal visits perspective. These works can be classified in two categories. The first category consists of task-based systems, these systems are generally designed for school visits. In these systems, the learner is supposed to accomplish various tasks in the museum with the support of a mobile device. The second category of systems is more designed for informal visits. In these systems, the visitor can browse among museum knowledge using the mobile device.

\subsection{Task Based Systems}

Museum Detective Guide [11] illustrates well task-oriented approaches. The system is intended for students, who interact with a mobile device. Students are grouped in pairs and the tour is imposed. In front of certain artworks, a series of multiple choice questions should encourage students to consider properly the work in question (e.g. What material is it made of?). Correct answers provide additional information while incorrect answers provide clues to determine the correct answer. In addition to traditional MCQ, learners may take part in more sophisticated games, such as drawing exercises. More open questions such as "What do you think the statue would say if it could talk?" aim at initiating discussion among learners about the exhibit. Several other task-based systems have been designed for mobile learning (not limited to the museum visit) CAESARUS [7], LORAMS [8], IPerC] (2008) ... Different kind of scenarios can be

\footnotetext{
${ }^{1} \mathrm{http}$ ///iperg.sics.se
} 
considered: role plays, simulations, puzzles ... One can find a review of such systems in [3]. Despite surface differences, these systems share various similarities. They share a constructivist perspective of learning, allowing the learner to construct representations through situated interactions. They all have a playful aspect and they promote social interaction between learners. These approaches are interesting because they help to maintain interest throughout the learning session, which is sometimes difficult for the younger students. However, in these approaches, the scenario and activities are highly constrained, leaving little effective freedom to learners. On the computer science perspective, these approaches are also limited. They are not generic and therefore not easily transferable to other locations or areas of learning.

\subsection{Navigation Based Systems}

Navigation-based systems differ from task-based system by only offering opportunities for browsing among documents related to work, without offering more sophisticated interactions (e.g. games, annotations). These approaches are rather intended to instrument informal visits of museum. Many navigation-based systems use knowledge representation formalisms to facilitate navigation through information resources (audio, video, etc.).

The HIPPIE project [9] was one of the first systems using automated reasoning in the context of museum visits. HIPPIE uses a characterization of works based on the ICONCLASS ${ }^{2}$ taxonomy, an exhaustive classification of the different themes of western art. Users are characterized by scores of interest for the different themes of the taxonomy. When an user moves into the museum, the system detects his position using a radio location technique. Therefore, HIPPIE is able to inform the user on the works around him that are relevant according to his interests. However, the artwork model in HIPPIE is based only on ICONCLASS), and HIPPIE can not propose self-assessments games.

The CHIP project [13] is a recommendation system of artworks based on the users' interests. The artwork model includes information from ICONCLASS and three artistic taxonomies published by the Getty Vocabularies Program ${ }^{3}$ ULAN (Union List of Artist Names), TGN (Thesaurus of Geographic Names) and AAT (Art and Architecture Thesaurus). When a user is interested by an artwork, he may give a score of interest to different characteristics of the work (style, subject, author ...). The system can then recommend him artworks that are also likely to be of interest (e.g. a visitor loving Magritte will be suggested surrealist painting). The artwork model of CHIP is richer than the artwork model of HIPPIE, however, it only allows comparison between artworks (not between artists for example), furthermore CHIP does not propose pedagogical activities.

These systems are interesting from the computer science perspective, as they are applicable to different museums and cultural venues. They are based on a semantic representation of works which gives them a certain genericity. In addition, they allow the user to have a large freedom during the visit and automatically adapt to his interests. However, they are poorly suited to support a visit by a class. They offer no educational activity and do not allow teachers to exercise guidance over the course of the visit. This work is at the intersection of these two approaches. It is based on a semantic model of the domain, that is to say, the cultural heritage. The use of semantic proximities permits to provide opportunities for navigation among museal knowledge. These proximities also allow the system to propose other types of activities such as self-assessment games about the artworks according to their characteristics. In addition, a semantic model of the visiting context is proposed, seamlessly supplied according to the movements of the visitor and his interaction with the application

\footnotetext{
${ }^{2}$ http://www.iconclass.nl

${ }^{3}$ http://www.getty.edu/research/tools/vocabularies
}

(e.g. consultation of information about an artwork, games ...). In comparison of the presented systems, this work is based on a rich semantic representation of artworks and on a generic semantic proximity computation. This genericness allows to compare each kind of instances of the knowledge base and to propose suggestions of artworks, authors, styles... when other systems are limited to suggestion of artworks. Moreover, as far as we know, unlike existing navigation-based systems, this work permits to propose relevant pedagogical activities while remaining generic.

\section{SEMANTIC MODELS OF DOMAIN AND CONTEXT}

\subsection{Existing Knowledge Resources for Cultural Heritage}

One of this work objectives is to help the visitor to understand the different types of links between the artworks. Therefore, a rich and precise representation of artworks is needed, that enables to highlight different aspects under which they can be compared. Considering the existing work in the field, the CIDOC-CRM ontology, the ICONCLASS (CLASSificication iconography) taxonomy and the Getty-AAT (Art and Architecture Thesaurus) thesaurus were selected for the representation of museum knowledge.

The CIDOC-CRM ontology is the reference ontology for semantic description of cultural heritage. It defines, among others, the concepts of event, work, person, place... using the RDFS knowledge description language. The CIDOC-CRM ontology is organized following a duality between physical objects, instances of CIDOC:PhysicalObject, with spatialization properties and temporal concepts (CIDOC:TemporalEntity) making it particularly suitable for the description of museum artifacts. A museum artifact can have a temporal extension accurately describing its creation mode, the activities in which it has been involved, it's successive owners... However, CIDOC-CRM is a very generic ontology which does not define concepts for artworks styles and themes. It therefore needs to be extended with the ICONCLASS and Getty-AAT controlled vocabularies.

ICONCLASS taxonomy permits to characterize the themes of the artworks. This is an exhaustive classification of artistic themes of Western art. The terms are organized as a hierarchy and there is no other relationship than Parent $\rightarrow$ Child relationship (i.e. no object property relations). This classification is extremely precise, for instance it exists a concept for "bearing the head, lifting ones hat" (which is a sub-concept of "salutating"). The Art and Architecture Thesaurus vocabulary is developed by the Getty Institute. It provides a controlled vocabulary for describing the qualities of the artworks and their mode of production. The thesaurus is organised into seven mains hierarchies or facets (e.g. Styles and Periods, Materials, Activities) with association and equivalence relation between the hierarchies. In the scope of this work only the Style and Periods hierarchy was used.

\subsection{Semantic Model of Artworks}

The CIDOC-CRM ontology is mainly focused on the concept of event, all physical entities, sub-concepts of CIDOC:PhysicalObject (e.g. person, artifact, building, collections) are connected to each others via temporal entities, subconcepts of CIDOC:TemporalEntity. Thus, there is no direct relationship to specify that an artist is the author of an artwork. To express such an assertion, it is necessary to associate the instance representing the artist with an instance of production event (CIDOC:Production) and associate the instance representing the artwork to the instance of the production event as the product of this production.

This kind of modelling, using an intermediate instance between two entities, is fairly standard. It is indeed possible to create com- 


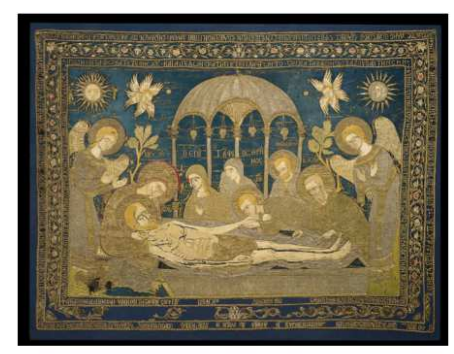

Epitaphios

Musée de Benaki

Athènes, Grèce

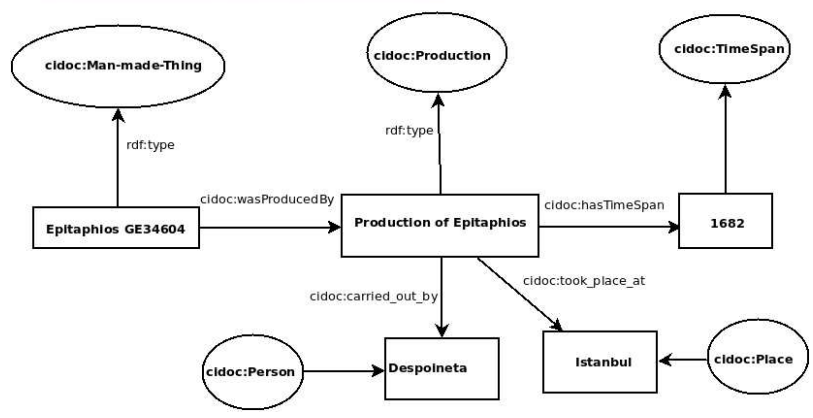

Fig. 1. Modelling of Epitaphios

plex relationships via the intermediate instance. For example the instance of event CIDOC:Production can be associated with a place (place of creation of the work) and a date (date of creation of the work) or to events have influenced the creation. This model is well suited in the case of museums and cultural heritage institutions. These institutions may wish, for example, to trace the sequence of events (e.g. legacies loans, seizures) which are involved in the history of a museum artifact. Figure 1 illustrates the modelling of the artwork Epitaphios (a religious tapestry of the seventeenth century) using the original model.

This model is rich, it is however relatively difficult to implement directly. Populating the knowledge base through the available sources of knowledge from the web of data is complex if one follows the original model. Indeed, the largest knowledge bases of the web of data (Freebase and DBPedia) are less rich in terms of expressivity than CIDOC-CRM. For example, the concept of producing a work does not exist in these bases. To populate the knowledge base following the CIDOC-CRM model, it is thus necessary to create an "artificial" instance of production event which will link the artist and the artwork. Moreover, as stated earlier, the purpose of this model is to allow computation of semantic proximities that will be intelligible to users. Several simplification of the CIDOC-CRM model were therefore needed. The objective of the simplifications of the model, in addition to facilitate the integration of external knowledge, is that these new properties will "speak" more to users when comparing two artworks. For example, instead of describing two painting as having close creation events, it is clearer to say them close regarding their creation dates.

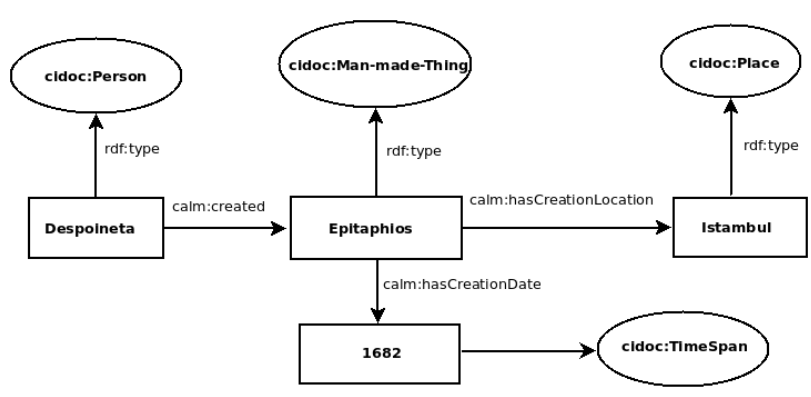

Fig. 2. Simplified modelling of Epitaphios
To that end, new properties were created by composition of others properties. That is to say some couples of triples of the form $\left(a, P_{i}, b\right)$ and $\left(b, P_{j}, c\right)$ were replaced by triples of the form $\left(a, P_{k}, c\right)$. The property $P_{k}$ being the composition of properties $P_{i}$ and $P_{j}$. These composition were applied in the case of properties involving temporal instances between physical objects. The result of these compositions applied to the modelling of Epitaphios is the simplified description presented in Figure 2.

In order to complete the semantic model of the domain, it was necessary to integrate into CIDOC-CRM the ICONCLASS hierarchy and the Getty-AAT thesaurus. Two problems arise for the integration of these controlled vocabularies in CIDOC-CRM: at first expressing these vocabularies in the form of RDFS taxonomy, then establishing links between these taxonomies and CIDOC-CRM.

To achieve this integration, these vocabularies were expressed in the SKOS formalism. The second step is to integrate these SKOS vocabularies in CIDOC-CRM. The purpose of this integration is to be able to make statements like: (CIDOC:LaJoconde calm:hasTheme iconClass:Portrait). The CIDOC-CRM ontology provides a specific concept for these kind of situations: the CIDOC:E55.Type concept. This concept is specifically designed to be an interface between controlled vocabularies and other concepts of CIDOC-CRM. Each element of ICONCLASS and Getty-AAT taxonomies is therefore an instance of CIDOC:E55.Type. Two sub-properties of CIDOC:hasType were also defined: calm:hasTheme and calm:hasStyle.

After integration of the controlled vocabularies, the semantic representation of artworks (instances of CIDOC:Man-MadeThing) is depicted in Figure 3.

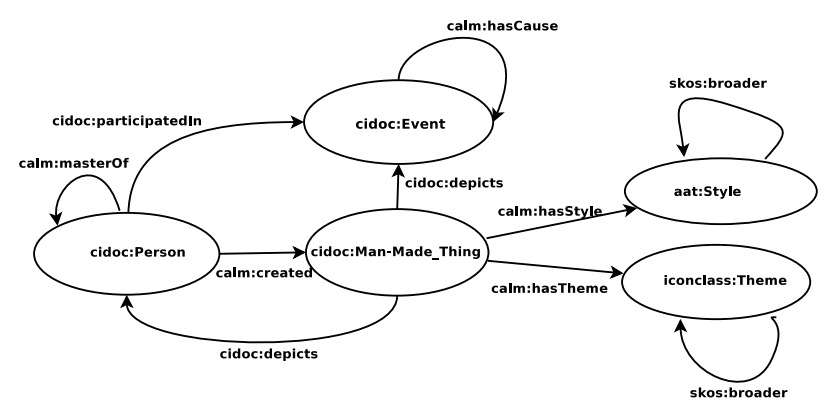

Fig. 3. Semantic model of artwork

\subsection{Semantic Model of Context}

The general principle of the context model is to represent the different kind of contexts (e.g. location, history of visit...) through different contextual spaces, each one structured by a specific domain ontology, for instance a space ontology for the location context. The domain ontology defines the kind of relationship between the visitor and the instances of the knowledge base museum. The domain ontology thus corresponds to the choice of a category of context, describing a modality of interaction between the visitor and artwork. The contextual spaces are populated with triples during explicit interaction between the visitor and the device (e.g. asking for more information about an artwork) or during implicit interactions (e.g. moving in the museum, stopping in front of an artwork).

Figure 4 explains this process: the visitor initiates an implicit (e.g. by stopping in front of an artwork) or explicit (e.g. by accessing to information about an artwork) interaction. This interaction triggers the rules for the population of contextual spaces labelled 1 and 2 in the example. Population rules use a domain ontology that defines the type of relationship between the visitor 


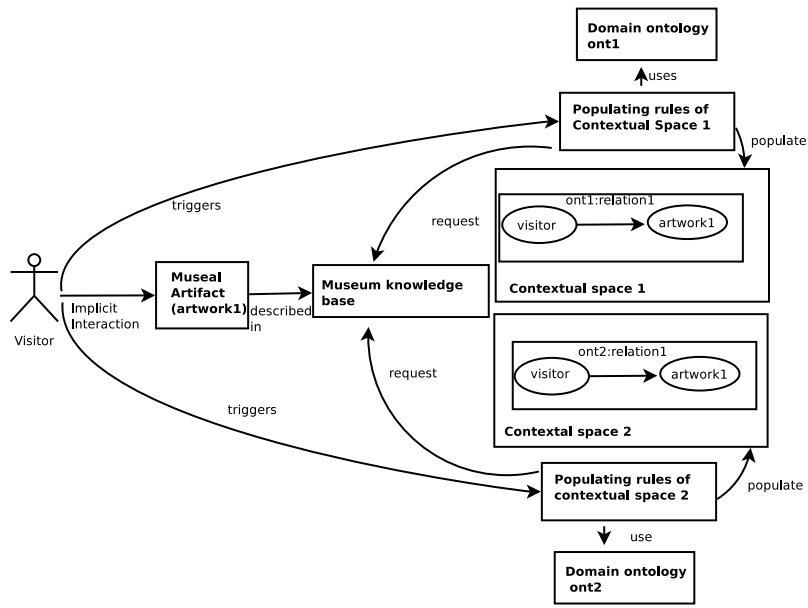

Fig. 4. Populating contextual spaces

and the artwork (e.g. contemplate the work, read the instructions ...) and add new statements to the contextual space.

Considering the works in the field of museology and museum learning, three contextual spaces were chosen to represent the context: the location context, the interaction history context and the interest context. These spaces are described extensively in [6]. The historical and interest contexts are structured by the SEM (Single Event Model) ontology [12]. Instances of the museal knowledge base corresponding to elements that have been consulted are added to the historical and interest contexts. The SEM ontology permits to give these elements a spatial and temporal extension (where and when items were consulted). The spatial context is based on the DaisY ontology, which is used to describe the physical space of the museum as well as the position of works.

\section{SEMANTIC AND CONTEXTUAL PROXIMITIES}

\subsection{Semantic Proximities}

One of this work objectives is to provide the user with relevant suggestions of paintings, artists, styles... whenever he consults information about a painting, an artist or a style. To allow this, it is necessary to have a numerical value that quantify the semantic proximity between two instances of concepts of the museum knowledge base.

To this end, the general approach of proximity by properties proposed by [10] was followed. However this approach have to be modified to suit to the specific situation of museum visit. The principle of this approach is to determine the proximity between two concepts by the properties they have in common. The more two concepts have properties in common, the more they can be considered close. However, the proximity proposed by [10] is applied to concepts of an ontology. In a museum visit situation, it is necessary to compute the proximity between instances of concepts. Indeed determining the proximity between the concept of Painting and the concept of Sculpture, for instance, would be of little interest for the user. Instead comparing a particular painting and a particular sculpture by trying to determine what they have in common provides the user interesting information. Rather than comparing the properties of concepts, the values of the properties of instances are compared.

The closer the values taken by the properties of two instances, the closer are these instances. The difficulty in the construction of this new measure of semantic proximity is the variety of value types of values type these properties can have. Properties may in fact have for range instances hierarchically organized (e.g. calm:hasStyle), instances not hierarchically organized (e.g. calm:hasParent) or datatype values (e.g. calm:hasBirthDate). Computation methods of proximity will differ according to the type of the range of the properties. Comparing two artists according to their styles will not be the same as comparing two artists according to their birth dates. The computation of proximity proposed here permits to integrate these heterogeneous information to construct a single numeric value.

To determine the set of properties of interest for visitors, the notion of concept of interest is defined. A concept of interest is a concept of the ontology which instances will appear in the proposed activities (e.g. suggestion, game...). A set of six concepts of interest were selected in the modified version of CIDOC-CRM ontology : Person, Style, Artwork, Historical Event, Place and Theme.

The choice of these concepts of interest permits to construct a matrix of properties of interest (Table 1 ). It is a matrix $T[i, j]$, where $T[0, k]=T[k, 0]$ are the concepts of interest and $T[i, j]$ are all relations in the ontology, with $T[i, 0]$ as domain and $T[0, j]$ as range. Thus, the line $i$ of the matrix defines the set of properties of interest that the concept $T[i, 0]$ have. The column $j$ of the matrix defines all the properties in which concept $T[0, j]$ is involved.

Table 1. Excerpt of matrix of properties of interest

\begin{tabular}{|l|l|l|l|}
\hline & cidoc:Person & getty:Style & cidoc:Artwork \\
\hline cidoc:Person & $\begin{array}{l}\text { [hasParent] } \\
\text { [hasMaster] } \\
\text { [hasStudent] }\end{array}$ & {$[$ hasStyle] } & $\begin{array}{l}\text { [partipatesIn cre- } \\
\text { ates] } \\
\text { [owns }]\end{array}$ \\
\hline getty:Style & {$[$ isStyleOf] } & $\begin{array}{l}\text { [broader] } \\
\text { [narrower }]\end{array}$ & {$\left[\begin{array}{l}\text { [isStyleOf] } \\
\end{array}\right.$} \\
\hline cidoc:Artwork & [depicts] & [hasStyle] & [partOf] \\
\hline
\end{tabular}

Line 2 of Table 1 permits to compare two instances of Cidoc:Person. Two persons can be compared according to their parents, teachers or students, styles and works they have created or they own. It is then possible to construct a vector of proximity quantifying, per property of interest, the proximity of the two instances.

Two methods of calculation for this proximity are used depending on the range of the property $P$. If the range of the property consists of concept which instances are not organized hierarchically (e.g. calm:hasMaster), the value of proximity is given by:

$$
\operatorname{Prox}_{P}(A, B)=\frac{|P(A) \cap P(B)|}{|P(A) \cup P(B)|}
$$

With $P(A)$ being the set of instances $i$ such as there exist a triple $(A, P, i) . \operatorname{Prox}_{P}(A, B)$ is the Jaccard index between the set of instances $P(A)$ and $P(B)$. This index is indeed appropriate to evaluate the proximity of two sets.

If the range of the property consists of concept which instances are organized hierarchically (e.g. calm:Style or calm:Theme), the value of the proximity is given by the proximity computation proposed by [14] between these two values:

$$
\operatorname{Prox}_{p}(A, B)=\frac{2 \cdot \operatorname{depth}(C)}{\operatorname{depth}_{C}(A)+\operatorname{depth}_{C}(B)}
$$

With $C$ being the least common subsummer of $A$ and $B$, depth $(C)$ the distance in arcs between $C$ and the root of the hierarchy and $\operatorname{depth}_{C}(A)$ the number of arcs between $A$ and the root of the hierarchy passing through $C$. This proximity is indeed well suited to hierarchies, its value depending both on the deepness of $A$ and $B$ in the hierarchy and in the number of arcs between them.

These computations permit to obtain a vector of proximity per property. In order to compare two instances depending on their 
proximity to a third instance, this vector must be aggregated in an unique value. To obtain a single value, it is assumed that all properties have the same importance. The value of the aggregation is the average of the values of the proximity per property vector. In the following $P(A, B)$ represents the value of the semantic proximity between instances $A$ and $B$.

The use of semantic proximity permits to provide two categories of contextualized activities in the museum. The first category concerns the recommendation of elements of interest to the visitor. In order to suggest elements of interest, the kind of element consulted by the user on his device is considered (e.g. artwork, biography, description of a theme ...). The visitor is then suggested to consider elements of the same kind (e.g. artwork, biography...) that are semantically close to the one he consults. For example, if the user consults the biography of Leonardo da Vinci, he may be suggested to consider the biography of Raphael, another painter of the Italian Renaissance. Furthermore, these suggestions are explained by sentences in natural language, generated using triples of the knowledge base used for the computation of proximity. For example, an argument to consider Raphael may be "He is another painter of the Italian Renaissance." The second category of activities are contextualized selfassessment games. Three types of games are proposed: MCQ (Multiple choice questions) games, true/false games and classification games (e.g. to classify paintings by date, style...). The generation process for MCQ is detailed below. From a statement of the knowledge base involving instances of concepts of interest, a question is generated. For example, the assertion (calm:deVinci calm:created calm:monaLisa) is transformed into the question "Who painted the Mona Lisa?". Distractors (incorrect answers) are then selected among the instances semantically close to the instance representing the right answer calm:deVinci.

\subsection{Contextual Proximities}

The use of semantic proximities for game or suggestion generation is interesting, but does not take into account what has been the actual tour of the visitor, the artworks he has seen, those around him... To provide truly personalized interactions the semantic proximities computations are completed by contextual proximities. These proximities permit to determine whether a game or a recommendation is consistent with the user's context.

The previous section presented how contextual spaces are populated during the visit. These spaces are primarily structures to represent museal knowledge "around" the user and in his interaction history. This section details how to compute contextual proximity between a game or a suggestion and the contextual spaces of the user.

Contextual proximity computation are very similar in the case of the historical context and location context. The computation for the location context is detailed here. The problem is to determine the proximity between a set $E$ of instances of the museum knowledge base (e.g. instances involved in a quiz or in a suggestion) and the location context of the user. To that end, the set $E_{L}$ is constructed which contains instances of the museum knowledge base that are present in the location context together with the values of their properties (for example, if a painting is present in the location context in $E_{L}$ the style and author of the painting are added in $E_{L}$ ).

Finally, the comparison between the set $E$ with the set $E_{L}$ is performed. This operation gives a contextual proximity value that quantifies the presence of instances of $E$ in the location context:

$$
\operatorname{Prox}_{\text {Context }}\left(E, E_{L}\right)=\frac{\left|E \cap E_{L}\right|}{\left|E_{L}\right|}
$$

If the value of $\operatorname{Prox}_{\text {Context }}$ is close to 1 , it means that the set $E$ is close to $E_{L}$. That is to say instances in $E$ are also present in the immediate environment of the user. For example, the instances associated with painters, styles and themes present in $E$ will be present around the visitor.

In the case of the interest context the computation is slightly different. The main idea is that if the visitor has an interest for a painter, he certainly have an interest for this painter artworks, his style... That is to say for the values of the properties of the instance representing the painter in question.

In order to take these interests into account, the set $E_{I}$ of the instances of the museum knowledge base present in the interest context is constructed and a semantic relaxation is applied to this set. That is to say, for each instance of the museum knowledge base in $E_{I}$, the values of properties of interest of this instance are added to the set. If $E_{r e l}$ denotes this new set, the proximity between a group of instances $E$ and the context of interest is computed as below:

$$
\operatorname{Prox}_{\text {Context }}\left(E, E_{I}\right)=\frac{\left|E \cap E_{I_{\text {rel }}}\right|}{\left|E_{I_{\text {rel }}}\right|}
$$

\subsection{Selection of Suggestion and Self-assessment Games to Propose}

To determine which suggestions to propose to the user, these suggestions are ordered according to their semantic and contextual proximities with the element that the user is considering (e.g. biography of an artist, explanation of an artwork). Let's suppose that the element in question is represented by the instance $I$ and one potential suggestion is represented by an instance $P$. A semantic relaxation is applied on this instance to obtain the set $P_{r e l}$. The principle of this relaxation is to add to the set $P_{r e l}$ the set of property interests of this instance and the instance $P$ itself.

The computation of the relevance score of the suggestion of an instance $P$ with respect to the instance $I$ is therefore:

$$
\begin{array}{r}
\operatorname{Score}\left(P, E_{I}, E_{L}, E_{H}\right)=\operatorname{ProxSem}(I, P)+ \\
\operatorname{Prox}_{\text {Context }}\left(P_{\text {rel }}, E_{I}, E_{L}, E_{H}\right)
\end{array}
$$

$\operatorname{Prox}_{\text {Context }}\left(P_{r e l}, E_{I}, E_{L}, E_{H}\right)$ being the mean of the sum of contextual proximities between $P_{r e l}$ and the history, location and interest contexts.

A very similar computation is used to determine the selfassessment games to propose to the user. Let's $G$ be a selfassessment game and $I(G)$ the set of instances involved in $G$ (for example the good answer and the distractors in the case of a MCQ game). The score of the game $G$ is given by:

$$
\begin{gathered}
\operatorname{Score}\left(I(G), E_{I}, E_{L}, E_{H}\right)= \\
\operatorname{Prox}_{\text {Context }}\left(I(G), E_{I}, E_{L}, E_{H}\right)
\end{gathered}
$$

\section{USAGE SCENARIO}

This section presents the developed prototype through an usage scenario. The user interacts directly with an Android client application. Computation of semantic and contextual proximities are realized by a server application which communicates with the client application via RestLet services. To illustrate the automatic population of contextual spaces during the use of the application, the states of the user's contexts are represented in the following screens.

The activities can be separated into two categories: consultation and navigation among museal knowledge (where the visitor is rather receiver) and self-assessment games (where the visitor is active). The visitor is free to switch whenever he wishes from one kind of activity to another. For the sake of simplicity, consultation and navigation activities are presented first following by the presentation of self-evaluation activities. 


\subsection{Browsing among Knowledge Associated to Artworks}

When the visit begins, the different contextual spaces (location, history and interest) are empty. The visitor moves through the museum space and when he encounters an artwork that interests him, he can use the device to select that artwork among the different artworks physically close to him (Figure 5). This explicit choice of the work is necessary due to the relatively poor precision of indoor localization tools.

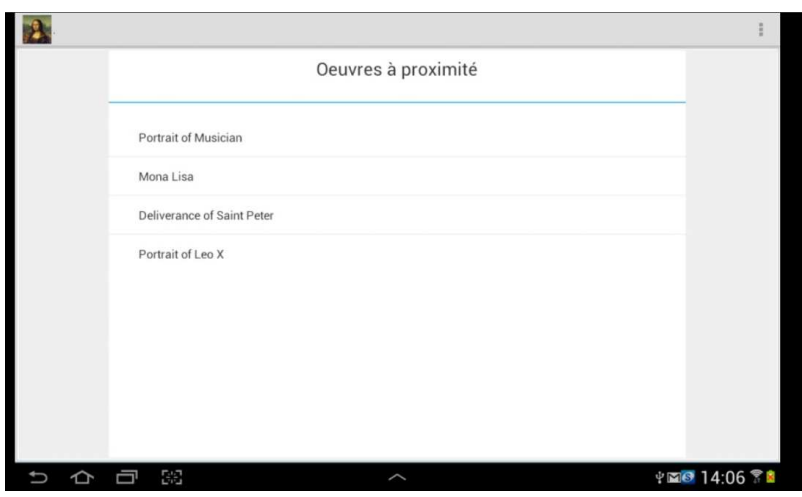

Fig. 5. Explicit selection of artwork

When the user has selected the artwork for which he wants information, he is directed to a page with information about that artwork (corresponding to the cartel, title, author and date of execution). The page also contains a more detailed description or the artwork. This description is not generated automatically (it is the same for all visitors). It corresponds to what can be find in the exhibition catalogue. Figure 6 presents the information page of The Lady with an Ermine by Leonardo da Vinci as well as the context state of the visitor. Since he is at the beginning of the visit, only the location context has elements.
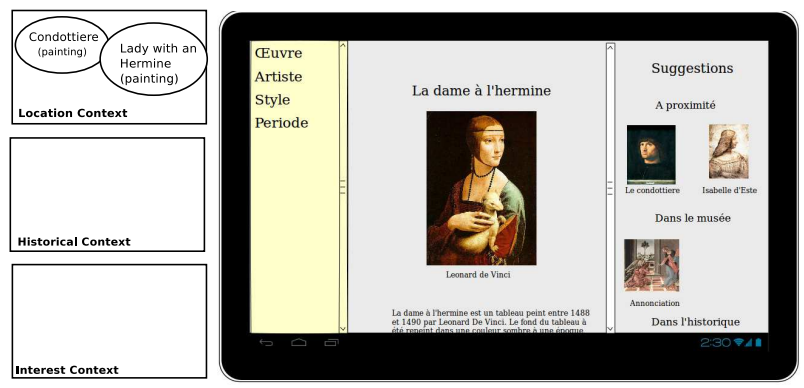

Fig. 6. Consultation of information about an artwork

The application screen of figure 6 has three parts. The left part, which will be discussed later, provides additional information related to the artwork. For instance, information about the artist, the style or the events depicted in the artwork.

The central part of the screen includes an image of the artwork to confirm to the user that he is consulting the information for the chosen artwork. This part has a vertical scroll bar to consult the full description.

Finally, the right part of the screen contains the suggestions proposed to the user. These suggestions are classified into three categories: the artworks in the museum that the user has not seen yet, the artworks that are close to the user (i.e. in his location context) and finally the artworks previously consulted by the user.
From this screen, several interactions are possible. At first, an implicit interaction: if the visitor stays long enough on the screen, the artwork is automatically added as part of his interest context. The visitor can also add this artwork to his interest context through the application menu. He may also consult the works that are suggested at the right of the screen. Figure 7 shows the consultation of the suggestion Le Condottiere. During the consultation of a suggestion, the justifications for this suggestion are exposed in natural language to the visitor. These justifications aim at helping the visitor to establish mental links between the different artworks.

\begin{tabular}{|c|c|c|c|}
\hline \multicolumn{4}{|l|}{ Suggestion } \\
\hline \begin{tabular}{|l|} 
Subject of \\
suggestion
\end{tabular} & Min score & Max score & Mean \\
\hline \begin{tabular}{|l|} 
Artist \\
\end{tabular} & 2 & 9 & 6,8 \\
\hline \begin{tabular}{|l|} 
Artwork \\
\end{tabular} & 1 & 10 & 7,2 \\
\hline \multicolumn{4}{|l|}{$\mathrm{MCQ}$} \\
\hline \begin{tabular}{|ll} 
Subject & of \\
MCQ & \\
\end{tabular} & Min score & Max score & Mean \\
\hline Artist & 3 & 8 & 7,1 \\
\hline Artwork & 4 & 9 & 7,7 \\
\hline
\end{tabular}

Fig. 7. Consultation of a suggestion

Finally, the user can also consult information about the author, the style, the characters, the period or events related to the artwork. To do this, he uses the left menu. Consultation of information about an entity associated to the artwork takes exactly the same form as the consultation of the artwork. Consultation of information about the author of The Lady with an Ermine is presented in Figure 8. Suggestions of artists semantically close to Leonardo da Vinci are proposed. When the visitor consults the suggestion of an artist, he also has access to a justification of that suggestion, which allows him to establish mental connection between the two artists.

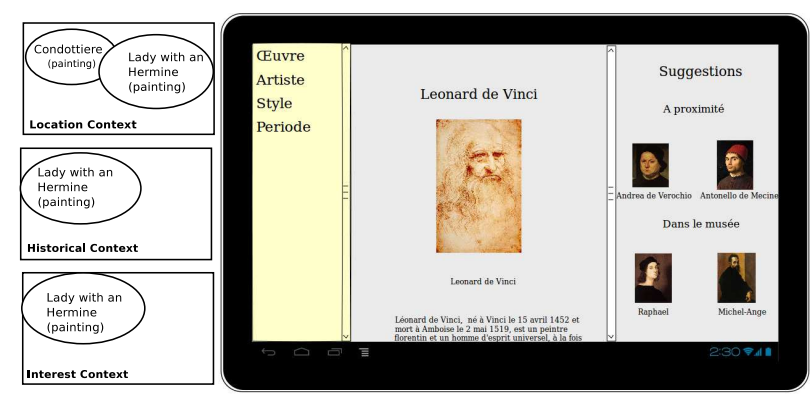

Fig. 8. Consultation of an artist biography

\subsection{Self-Assessment Games}

At any time during the visit, the visitor may access to selfassessment games. These activities aim at providing a relaxing playtime during the visit and to allow visitors to validate their knowledge through exercises of increasing difficulty.

When the user selects the item Go to games in the application menu, he is directed to the MCQ games page. One question, in line with its context as described in Section 3, is then proposed (figure 9).

Here again several options are available to the user: he may of course answer the question. In this case a message will inform him if the answer is correct or incorrect by giving the correct 


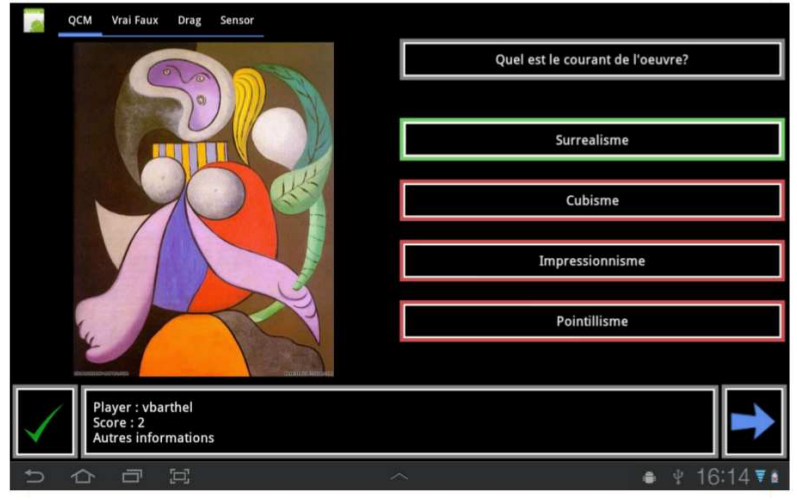

Fig. 9. Example of an MCQ game

answer if necessary. The user may also, load a different question with the Reload button at the bottom right of the screen. Other games are accessible (Figure 10) via a horizontal scrolling, fairly standard for this type of application.

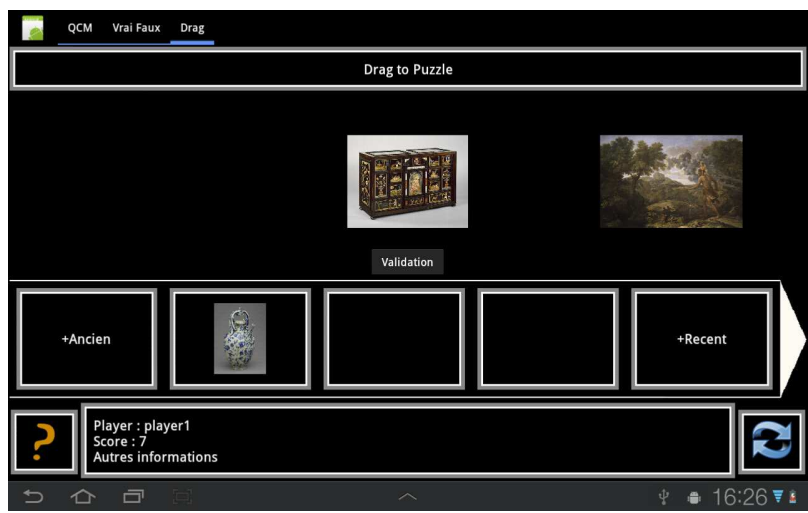

Fig. 10. Example of classification game

\section{VALIDATION}

Two main aspects of this work were evaluated: the relevance of the generated questions and recommendations, and the usability of the system in a museum visit situation.

\subsection{Relevance of Generated Questions and Recommendations}

To assess the relevance of the questions and recommendations generated by the system, a knowledge base containing fifty works and ten artists from the Middle Ages to modern times was built. That knowledge base was generated from semantic descriptions available in DBPedia. For each work and artist of the knowledge base, the four closest recommendations were generated together with a set of MCQ about artists and artworks. A domain expert was then asked to assign a score from 0 to 10 for each set of recommendations and a score from 0 to 10 for each question. The results are summarized in the figure 11.

The average score is relatively good for recommendations and questions. The low minimum can be explained by the mode of construction of the knowledge base. Artists and works with low scores in terms of recommendation or questions are artists or works whose semantic description is extremely brief in DBpedia. These scores may be improved if the descriptions are completed.

\begin{tabular}{|l|c|c|c|}
\hline \begin{tabular}{l} 
Suggestion \\
\hline $\begin{array}{l}\text { Subject of } \\
\text { suggestion }\end{array}$
\end{tabular} & Min score & Max score & Mean \\
\hline Artist & 2 & 9 & 6,8 \\
\hline Artwork & 1 & 10 & 7,2 \\
\hline \multicolumn{1}{|c|}{ MCQ } & \multicolumn{3}{|l|}{} \\
\hline $\begin{array}{l}\text { Subject of } \\
\text { MCQ }\end{array}$ & Min score & Max score & Mean \\
\hline Artist & 3 & 8 & 7,1 \\
\hline Artwork & 4 & 9 & 7,7 \\
\hline
\end{tabular}

Fig. 11. Evaluation of recommendations and MCQ

\subsection{Usability experiment}

An usability experiment was conducted during an actual museum visit. The experiment took place in the Great Hall of the Imperial Palace of Compiegne (France) and involved 37 visitors. The classical approach for interfaces evaluation, System Usability Scale method [2] was used. The visitors were asked to perform a set of tasks using the application: consultation of information about artworks, consultation of suggestion, free annotation of artworks and consultation of annotations. The visitors then filled a questionnaire to assess the usability and usefulness of each task. The table 2 summarizes the scores of usability and usefulness of different tasks on a scale from 0 to 100 .

Table 2. Usability and utility

\begin{tabular}{|l|c|c|}
\hline Task & Usability & Utility \\
\hline Consult artwork description & 83 & 97 \\
\hline Consult suggestion & 78 & 86 \\
\hline Consult annotation & 73 & 60 \\
\hline Submit an annotation & 63 & 52 \\
\hline
\end{tabular}

The consultation and suggestion activities achieve good scores in terms of usability and usefulness. The tasks getting the worst scores are free annotations of artworks and consultation of annotations. Following this experiment, free annotation were completed with self-assessment games which appears to be more suitable in the context of an informal museum visit.

\section{CONCLUSION}

This paper presented an ubiquitous learning system designed to assist informal museum visits. Using a semantic representation of the context and cultural heritage various contextualized activities were proposed to help visitors to navigate through the museum knowledge and to use the acquired knowledge through selfassessment activities. The originality of this proposal is based on the dual modelling, semantic and contextual, which permits to provide activities that are both semantically and contextually relevant to the visitor.

\section{REFERENCES}

[1] David Anderson, Keith B. Lucas, and Ian S. Ginns. Theoretical perspectives on learning in an informal setting. Journal of Research in Science Teaching, 40(2):177-199, February 2003.

[2] J. Brooke, P. W. Jordan, B. Weerdmeester, A. Thomas, and I. L. Mclelland. Usability evaluation in industry. SUS: A quick and dirty usability scale, 1996.

[3] A. Damala. Interaction Design and Evaluation of Mobile Guides for the Museum Visit: A Case Study in Multimedia and Mobile Augmented Reality. PhD Thesis. $\mathrm{PhD}$ thesis, CEDRIC Laboratory, Paris, France, 2009.

[4] S. Debenedetti. Visite occasionnelle du musée d'art et confort de visite: le role des compagnons. 2003. 
[5] John Falk. The director's cut: Toward an improved understanding of learning from museums. Science Education, 88(S1):S83S96, 2004.

[6] P.-Y. Gicquel and D. Lenne. Un modèle sémantique et contextuel pour l'apprentissage informel. Revue d'Intelligence Artificielle, 26(3):309-338, 2012.

[7] L. Naismith, M. Sharples, and J. Ting. Evaluation of CAERUS: a context aware mobile guide. H. van der Merwe \& T. Brown, Mobile Technology: The Future of Learning in Your Hands, page 50, 2005.

[8] Hiroaki Ogata, Yoshiki Matsuka, Moushir El-bishouty, and Yoneo Yano. LORAMS: capturing, sharing and reusing experiences by linking physical objects and videos. 2008.

[9] R. Oppermann and M. Specht. A nomadic information system for adaptive exhibition guidance. Archives and museum informatics, 13(2):127138, 1999.

[10] G. Pirro and J. Euzenat. A feature and information theoretic framework for semantic similarity and relatedness. The Semantic Web ISWC 2010, pages 615-630, 2010.
[11] J. Thom-Santelli, C. Toma, K. Boehner, and G. Gay. Beyond just the facts: Museum detective guides. HCI Group, Cornell University. Retrieved January, 26:2006, 2005.

[12] W.R. van Hage, V. Malais, R. Segers, L. Hollink, and G. Schreiber. Design and use of the simple event model (SEM). Web Semantics: Science, Services and Agents on the World Wide Web, 2011.

[13] Y. Wang, N. Stash, L. Aroyo, L. Hollink, and G. Schreiber. Using semantic relations for content-based recommender systems in cultural heritage. In Proceedings of the Workshop on Ontology Patterns (WOP) at ISWC, pages 16-28, 2009.

[14] Z. Wu and M. Palmer. Verbs semantics and lexical selection. In Proceedings of the 32nd annual meeting on Association for Computational Linguistics, pages 133-138, 1994.

[15] Andreas Zimmermann, Andreas Lorenz, and Reinhard Oppermann. An operational definition of context. In Proceedings of the 6th international CONTEXT conference, pages 558-571. Springer-Verlag, 2007. 